\title{
Contributions to the Macromycota of Gaziantep Province
}

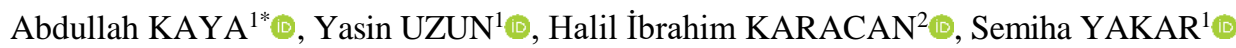 \\ ${ }^{1}$ Karamanoğlu Mehmetbey University, Science Faculty, Department of Biology, Karaman, TURKEY \\ ${ }^{2}$ Ömer Özmimar Religious Anatolian High School, Gaziantep, TURKEY \\ *Corresponding author: kayaabd@ hotmail.com
}

Received Date: 02.01.2019

Accepted Date: 25.09.2019

\begin{abstract}
Aim of study: The study aims to determine the macrofungal biodiversity of Gaziantep province and to contribute to the mycobiota of Turkey.

Area of study: The study was carried out in Gaziantep province which is located in Southeastern Anatolian Region of Turkey. The study area takes place at the transition region of Mediterranean and IranoTuranean phytogeographic regions and falls in C6 according to Davis' grid square system.

Material and method: The fungal specimens were collected from different localities within the boundaries of Gaziantep province between 2009 and 2015. After they were photographed at their natural habitats, they were transferred to the fungarium and the descriptive data related to their macroscopy and microscopy were obtained. Then the samples were identified by comparing the obtained descriptive data with literature data.

Main results: Besides the taxa reported from the region before, 217 macrofungi species belonging to 129 genera, 63 families, 19 orders and 6 classes within Ascomycota and Basidiomycota were determined from Gaziantep.

Research highlights: The mycota of Turkey has not prepared yet. That is why biodiversity studies on the macromycota of Turkey is an important requirement to present the overall macrofungal diversity of the country.

Keywords: Biodiversity, Macrofungi, Gaziantep, Turkey
\end{abstract}

\section{Gaziantep Makromikotasına Katkılar}

Öz

Çalışmanın amacı: Bu çalışma Gaziantep'in makromantar biyoçeşitliliğini belirlemeyi ve Türkiye mikobiyotasına katkı sağlamayı amaçlamaktadır.

Çalışma alanı: Çalışma, Türkiye'nin Güneydoğu Anadolu Bölgesi'nde yer alan Gaziantep'te gerçekleştirilmiştir. Çalışma alanı, Akdeniz ve İran-Turan fitocoğrafik bölgelerinin geçiş bölgesinde ve Davis'in kareleme sistemine gore C6 karesi içinde yer almaktadır.

Materyal ve yöntem: Makromantar örnekleri 2009-2015 yılları arasında Gaziantep sınırları içinde kalan farklı lokalitelerden toplanmıştır. Doğal ortamlarında fotoğraflanan örnekler fungaryuma taşınarak makroskobi ve mikroskobilerine yönelik betimleyici data elde edilmiştir. Derlenen betimleyici data literatür verileriyle karşılaştırılarak örneklerin teşhisleri yapılmıştır.

Temel Sonuçlar: Bölgeden daha önce verilen taksonların dişında, Gaziantep’ten Ascomycota ve Basidiomycota bölümleri içinde yer alan 6 sınıf, 19 ordo, 63 familya ve 129 cinse ait 217 makromantar türü belirlenmiştir.

Araştırma vurguları: Türkiye mikotosı henüz hazırlanmamıştır. Bu nedenle, ülkenin bütüncül makromantar kompozisyonunun ortaya konulabilmesi amacıyla Türkiye makromantarları ile ilgili biyoçeşitlilik çalışmalarının yapılması önemli bir gerekliliktir.

Anahtar kelimeler: Biyoçeşitlilik, Makromantarlar, Gaziantep, Türkiye 


\section{Introduction}

The number of taxonomic studies on the macrofungi of Turkey has increased especially in the last three to four decades. About 2400 macromycete taxa have been presented in checklists prepared by Sesli and Denchev (2014) and Solak, Iş̧loğlu, Kalmış and Allı (2015). Additional contributions were also made to these lists by Kaya (2015), Demirel, Uzun, Keleş, Akçay and Acar (2017), Işık and Türkekul (2017), Kaşık, Aktaş, Alkan and Öztürk (2017), Öztürk, Pamukçu and Aktaş (2017), Türkekul and Işık (2017), Sesli (2018), Tırpan, Çöl, Şen and Allı (2018) and Şelem, Keleş, Acar and Demirel (2019). Compared to the plant diversity of Turkey and 15.000 macrofungi taxa, reported from Europe (Lukić, 2009), there is still much to be done to obtain the overall macrofungal data of Turkey.

Gaziantep is situated in Southeastern Anatolian region of Turkey and bordered by Kahramanmaraş to the north, the river Euphrates to the east, Kilis and Syria to the South and Osmaniye to the west. The area falls in Irano-Turanian phytogeographical sector within the holoarctic floral kingdom and has a Mediterranean climate (Akman, 1999) with an annual average temperature of $14.9^{\circ} \mathrm{C}$ and annual rainfall of $\sim 565 \mathrm{~mm}$. Forest areas are characterized mainly by Quercus L. and planted Pinus brutia Ten. populations. Members of the genera Pistacia, Paliurus, Morus, Crataegus, Amygdalus and Rosa are some other representative tree populations in the region. At higher elevations, especially on Amanos mountain range, Abies cilicica subsp. cilicica, Pinus nigra subsp. pallasiana, Fagus orientalis, Carpinus orientalis, Taxus baccata, Alnus glutinosa subsp. glutinosa, Corylus avellana var. avellana also have specific distributions (İskender et al., 2005). Various species of Salix, Populus, Platanus and Tamarix are dominant along the river and stream banks.

Starting from 2009 some local lists and 95 new records were presented from Gaziantep province (Kaya \& Uzun, 2015; Uzun, Kaya, Karacan, Kaya \& Yakar, 2015; Kaya, Uzun, Karacan \& Yakar, 2016; Uzun, Karacan, Yakar \& Kaya, 2018a; Uzun, Yakar, Karacan \& Kaya, 2018b).
The current study was based on macrofungi specimens collected from the rest of the province, and aims to determine the macrofungal biodiversity of the region and to contribute to the knowledge of the mycobiota of Turkey.

\section{Materials and Methods}

Macrofungi samples were collected from 64 localities (Table 1) within the boundaries of Gaziantep Province between 2009 and 2015. Required field data were recorded and the specimens were photographed in their natural habitats. Necessary macroscopic and microscopic investigations were carried out in the fungarium. The specimens were identified by comparing the obtained data with Phillips (1981), Breitenbach and Kränzlin (19842000), Miller and Miller (1988), Bessette, Bessente and Fischer (1997), Bessente, Roody and Bessente (2007), Ellis and Ellis (1990), Ellis and Ellis (1997), Hansen and Pfister (2006), Medardi (2006) and Beug, Bessente and Bessente (2014). Fruit bodies are kept at Karamanoğlu Mehmetbey University, Science Faculty, Karaman, Turkey.

\section{Results}

The systematics of the taxa are given in accordance with Kirk, Cannon, Minter and Stalpers (2008) and Index Fungorum (2018).

Ascomycota Caval.-Sm.

Dothideomycetes O.E. Erikss. \& Winka Patellariales D. Hawksw. \& O.E. Erikss. Patellariaceae Corda

1. Patellaria atrata (Hedw.) Fr.: Mixed forest, on Quercus sp. twigs, locality 36, 05.12.2014, K.10893.

Leotiomycetes O.E. Erikss. \& Winka

Helotiales Nannf.

Dermateaceae Fr.

2. Mollisia cinerea (Batsch) P. Karst.: On Quercus sp. branch, locality 64, 13.04.2013, K.7906.

3. Mollisia ligni (Desm.) P. Karst.: On Quercus sp. twigs, locality 37, 10.04.2015, K.11683.

4. Mollisia melaleuca (Fr.) Sacc.: On Quercus sp. twigs, locality 10, 02.05.2015, K.11868.

Helotiaceae Rehm 
5. Ascocoryne cylichnium (Tul.) Korf: On Fagus sp. twig, locality 12, 19.10.2014, K. 10080.
6. Cenangium ferruginosum Fr.: On dead Pinus sp. bark, locality 36, 04.04.2015, K.11598.

Table 1. Macrofungi collection localities

\begin{tabular}{|c|c|c|c|}
\hline No & Localities & Coordinates & Altitudes (m) \\
\hline 1 & Araban, Emirhaydar & $37^{\circ} 30^{\prime} \mathrm{N}-37^{\circ} 42 \mathrm{E}$ & 850 \\
\hline 2 & Araban, Eskialtuntaş & $37^{\circ} 24^{\prime} \mathrm{N}-37^{\circ} 41 \mathrm{E}$ & 513 \\
\hline 3 & İslahiye, Alaca & $36^{\circ} 59^{\prime} \mathrm{N}-36^{\circ} 49^{\prime} \mathrm{E}$ & 1020 \\
\hline 4 & İslahiye, Altınüzüm & $36^{\circ} 54^{\prime} \mathrm{N}-36^{\circ} 34^{\prime} \mathrm{E}$ & 490 \\
\hline 5 & İslahiye, Çubuk & $36^{\circ} 59^{\prime} \mathrm{N}-36^{\circ} 48^{\prime} \mathrm{E}$ & 800 \\
\hline 6 & İslahiye, Fevzipaşa & $37^{\circ} 06^{\prime} \mathrm{N}-36^{\circ} 37^{\prime} \mathrm{E}$ & 675 \\
\hline 7 & İslahiye, Hanağzı & $37^{\circ} 03^{\prime} \mathrm{N}-36^{\circ} 36^{\prime} \mathrm{E}$ & 535 \\
\hline 8 & İslahiye, Hasanlök & $36^{\circ} 54^{\prime} \mathrm{N}-36^{\circ} 30^{\prime} \mathrm{E}$ & 815 \\
\hline 9 & İslahiye, Kozdere & $37^{\circ} 06^{\prime} \mathrm{N}-36^{\circ} 39^{\prime} \mathrm{E}$ & 750 \\
\hline 10 & İslahiye, Tandır & $36^{\circ} 57^{\prime} \mathrm{N}-37^{\circ} 31^{\prime} \mathrm{E}$ & 695 \\
\hline 11 & İslahiye, Tandır & $36^{\circ} 58^{\prime} \mathrm{N}-36^{\circ} 29^{\prime} \mathrm{E}$ & 1750 \\
\hline 12 & İslahiye, Tandır & $36^{\circ} 57^{\prime} \mathrm{N}-37^{\circ} 29^{\prime} \mathrm{E}$ & 1530 \\
\hline 13 & Karkamış, Gürçay & $36^{\circ} 53^{\prime} \mathrm{N}-38^{\circ} 01^{\prime} \mathrm{E}$ & 340 \\
\hline 14 & Karkamış, Kelekli & $36^{\circ} 55^{\prime} \mathrm{N}-37^{\circ} 59^{\prime} \mathrm{E}$ & 340 \\
\hline 15 & Karkamış, Yurtbağı & $36^{\circ} 50^{\prime} \mathrm{N}-38^{\circ} 00^{\prime} \mathrm{E}$ & 330 \\
\hline 16 & Nizip, Gökçeli & $36^{\circ} 54^{\prime} \mathrm{N}-37^{\circ} 43^{\prime} \mathrm{E}$ & 650 \\
\hline 17 & Nizip, Mercanlı & $36^{\circ} 56^{\prime} \mathrm{N}-37^{\circ} 46^{\prime} \mathrm{E}$ & 493 \\
\hline 18 & Nizip, Sekili & $36^{\circ} 58^{\prime} \mathrm{N}-37^{\circ} 40^{\prime} \mathrm{E}$ & 600 \\
\hline 19 & Nurdağı, Ataköy & $37^{\circ} 08^{\prime} \mathrm{N}-36^{\circ} 54^{\prime} \mathrm{E}$ & 985 \\
\hline 20 & Nurdağı, Atmalı & $37^{\circ} 07^{\prime} \mathrm{N}-36^{\circ} 53^{\prime} \mathrm{E}$ & 700 \\
\hline 21 & Nurdağı, Atmalı & $37^{\circ} 08^{\prime} \mathrm{N}-36^{\circ} 52^{\prime} \mathrm{E}$ & 620 \\
\hline 22 & Nurdağı, Atmalı & $37^{\circ} 07^{\prime} \mathrm{N}-36^{\circ} 53^{\prime} \mathrm{E}$ & 700 \\
\hline 23 & Nurdağı, Atmalı & $37^{\circ} 07^{\prime} \mathrm{N}-36^{\circ} 51^{\prime} \mathrm{E}$ & 715 \\
\hline 24 & Nurdağı, Bademli & $37^{\circ} 13^{\prime} \mathrm{N}-36^{\circ} 45^{\prime} \mathrm{E}$ & 610 \\
\hline 25 & Nurdağı, Belpınar & $37^{\circ} 12^{\prime} \mathrm{N}-36^{\circ} 46^{\prime} \mathrm{E}$ & 540 \\
\hline 26 & Nurdağı, Belpınar & $37^{\circ} 12^{\prime} \mathrm{N}-36^{\circ} 45^{\prime} \mathrm{E}$ & 545 \\
\hline 27 & Nurdağı, Hamidiye & $37^{\circ} 06^{\prime} \mathrm{N}-36^{\circ} 53^{\prime} \mathrm{E}$ & 810 \\
\hline 28 & Nurdağı, Hamidiye & $37^{\circ} 05^{\prime} \mathrm{N}-36^{\circ} 53^{\prime} \mathrm{E}$ & 800 \\
\hline 29 & Nurdağı, İçerisu & $37^{\circ} 09^{\prime} \mathrm{N}-36^{\circ} 50^{\prime} \mathrm{E}$ & 580 \\
\hline 30 & Nurdağı, İncirli & $37^{\circ} 14^{\prime} \mathrm{N}-36^{\circ} 59^{\prime} \mathrm{E}$ & 600 \\
\hline 31 & Nurdağı, Kırkpınar & $37^{\circ} 03^{\prime} \mathrm{N}-36^{\circ} 51^{\prime} \mathrm{E}$ & 920 \\
\hline 32 & Nurdağı, Kırkpınar & $37^{\circ} 04^{\prime} \mathrm{N}-36^{\circ} 52^{\prime} \mathrm{E}$ & 975 \\
\hline 33 & Nurdağı, Kömürler & $37^{\circ} 10^{\prime} \mathrm{N}-36^{\circ} 48^{\prime} \mathrm{E}$ & 910 \\
\hline 34 & Nurdağı, Kömürler & $37^{\circ} 09^{\prime} \mathrm{N}-36^{\circ} 48^{\prime} \mathrm{E}$ & 535 \\
\hline 35 & Nurdağı, Kuzoluk & $36^{\circ} 04^{\prime} \mathrm{N}-36^{\circ} 54^{\prime} \mathrm{E}$ & 1200 \\
\hline 36 & Nurdağı, Olucak & $37^{\circ} 10^{\prime} \mathrm{N}-36^{\circ} 41^{\prime} \mathrm{E}$ & 810 \\
\hline 37 & Nurdağı, Olucak & $37^{\circ} 10^{\prime} \mathrm{N}-36^{\circ} 40^{\prime} \mathrm{E}$ & 950 \\
\hline 38 & Nurdağı, Olucak & $37^{\circ} 09^{\prime} \mathrm{N}-36^{\circ} 40^{\prime} \mathrm{E}$ & 980 \\
\hline 39 & Nurdağı, Ökkeşiye & $37^{\circ} 04^{\prime} \mathrm{N}-37^{\circ} 50^{\prime} \mathrm{E}$ & 1010 \\
\hline 40 & Nurdağı, Sakçagöz & $37^{\circ} 10^{\prime} \mathrm{N}-36^{\circ} 55^{\prime} \mathrm{E}$ & 850 \\
\hline 41 & Nurdağı, Tüllüce & $37^{\circ} 09^{\prime} \mathrm{N}-36^{\circ} 51^{\prime} \mathrm{E}$ & 590 \\
\hline 42 & Oğuzeli, centre & $36^{\circ} 58^{\prime} \mathrm{N}-37^{\circ} 30^{\prime} \mathrm{E}$ & 700 \\
\hline 43 & Oğuzeli, centre & $36^{\circ} 57^{\prime} \mathrm{N}-37^{\circ} 30^{\prime} \mathrm{E}$ & 670 \\
\hline 44 & Oğuzeli, Karataş & $36^{\circ} 53^{\prime} \mathrm{N}-37^{\circ} 33^{\prime} \mathrm{E}$ & 627 \\
\hline 45 & Oğuzeli, Yakacık & $36^{\circ} 54^{\prime} \mathrm{N}-37^{\circ} 32^{\prime} \mathrm{E}$ & 639 \\
\hline 46 & Oğuzeli, Yalnızbağ & $36^{\circ} 59^{\prime} \mathrm{N}-37^{\circ} 26^{\prime} \mathrm{E}$ & 782 \\
\hline 47 & Şahinbey, Aslanlıbel & $37^{\circ} 01^{\prime} \mathrm{N}-37^{\circ} 22^{\prime} \mathrm{E}$ & 840 \\
\hline 48 & Şahinbey, centre & $37^{\circ} 03^{\prime} \mathrm{N}-37^{\circ} 21^{\prime} \mathrm{E}$ & 865 \\
\hline 49 & Şahinbey, Gaziantep Zoo & $37^{\circ} 02^{\prime} \mathrm{N}-37^{\circ} 17^{\prime} \mathrm{E}$ & 970 \\
\hline 50 & Şahinbey, Öğümsöğüt & $37^{\circ} 07^{\prime} \mathrm{N}-37^{\circ} 17^{\prime} \mathrm{E}$ & 1060 \\
\hline
\end{tabular}


Table 1. (continued)

\begin{tabular}{llcc}
\hline No & Localities & Coordinates & Altitudes (m) \\
\hline 51 & Şahinbey, Yeşilkent & $37^{\circ} 02^{\prime} \mathrm{N}-37^{\circ} 25^{\prime} \mathrm{E}$ & 780 \\
52 & Şehitkamil, Atalar & $37^{\circ} 11^{\prime} \mathrm{N}-36^{\circ} 01^{\prime} \mathrm{E}$ & 1140 \\
53 & Şehitkamil, Bus station & $37^{\circ} 06^{\prime} \mathrm{N}-37^{\circ} 24 \mathrm{E}$ & 840 \\
54 & Şehitkamil, Cerityeniyapan & $37^{\circ} 10^{\prime} \mathrm{N}-37^{\circ} 08^{\prime} \mathrm{E}$ & 1070 \\
55 & Şehitkamil, Durnalık & $37^{\circ} 10^{\prime} \mathrm{N}-37^{\circ} 11^{\prime} \mathrm{E}$ & 1000 \\
\hline 56 & Şehitkamil, Dülükbaba picnic area & $37^{\circ} 06^{\prime} \mathrm{N}-37^{\circ} 19^{\prime} \mathrm{E}$ & 1040 \\
57 & Şehitkamil, Dülükbaba picnic area & $37^{\circ} 07^{\prime} \mathrm{N}-37^{\circ} 19^{\prime} \mathrm{E}$ & 1110 \\
58 & Şehitkamil, Yeşilce & $37^{\circ} 10^{\prime} \mathrm{N}-37^{\circ} 12^{\prime} \mathrm{E}$ & 1045 \\
59 & Yavuzeli, Aşağlkekliktepe & $37^{\circ} 17^{\prime} \mathrm{N}-37^{\circ} 36^{\prime} \mathrm{E}$ & 830 \\
60 & Yavuzeli, Büyükkarakuyu & $37^{\circ} 13^{\prime} \mathrm{N}-37^{\circ} 31^{\prime} \mathrm{E}$ & 600 \\
61 & Yavuzeli, Çimenli & $37^{\circ} 16^{\prime} \mathrm{N}-37^{\circ} 34^{\prime} \mathrm{E}$ & 710 \\
62 & Yavuzeli, Halilbaşlı & $37^{\circ} 16^{\prime} \mathrm{N}-37^{\circ} 31^{\prime} \mathrm{E}$ & 560 \\
63 & Yavuzeli, Küçükkarakuyu & $37^{\circ} 21^{\prime} \mathrm{N}-37^{\circ} 33^{\prime} \mathrm{E}$ & 980 \\
64 & Yavuzeli, Sarıbuğdaylı & $37^{\circ} 17^{\prime} \mathrm{N}-37^{\circ} 31^{\prime} \mathrm{E}$ & 560 \\
\hline
\end{tabular}

7. Hymenoscyphus calyculus (Fr.) W. Phillips: On Fagus sp. twig, locality 11, 01.11.2014, K.10452.

8. Hymenoscyphus fructigenus (Bull.) Gray: On decaying Quercus sp. cupules, locality 62, $02.11 .2014, \quad$ K. 10468; locality 39, 07.11.2015, K.12720.

9. Hymenoscyphus herbarum (Pers.) Dennis: On dead herb stem, locality 50, 16.11.2014, K.10683; locality 42, 13.12.2014, K.11068.

10. Hymenoscyphus serotinus (Pers.) W. Phillips: On Fagus sp. twig, locality 11, 14.11.2015, K.12781.

Marthamycetaceae H.O. Baral, G. Lantz, Hustad \& Minter

11. Naemacyclus fimbriatus (Schwein.) DiCosmo, Peredo \& Minter: On pine cones, locality 8, 07.03.2014, K.8532.

Rutstroemiaceae Holst-Jensen

12. Rutstroemia firma (Pers.) P. Karst.: On pine twigs, locality 36, 05.12.2014, K.10926.

Sclerotiniaceae Whetzel

13. Ciboria rufofusca (O. Weberb.) Sacc.: On dead Abies sp. cones, locality 11, 26.04.2014, K.8877.

14. Sclerotinia sclerotiorum (Lib.) de Bary: On soil under Populus sp., locality 62, 29.03.2015, K.11558.

Rhytismatales M.E. Barr ex Minter

Rhytismataceae Chevall.

15. Lophodermium

arundinaceum

(Schrad.) Chevall.: On pine needles, locality 30, 05.12.2014, K.10877.
16. Propolis farinosa (Pers.) Fr.: On Quercus sp. twigs, locality 36, 05.12.2014, K.10892; locality 39, 12.04.2015, K.11743.

Pezizomycetes O.E. Erikss. \& Winka

Pezizales J. Schröt.

Caloscyphaceae Harmaja

17. Caloscypha fulgens (Pers.) Boud.: Among needle litter, locality 37, 29.03.2013, K.7808.

Helvellaceae Fr.

18. Helvella acetabulum (L.) Quél.: Among leaf litter in mixed forest, locality 40, 18.04.2015, K.11763; locality 31, 18.04.2015, K.11768.

19. Helvella lacunosa Afzel.: Among grass in mixed forest, locality 36, 10.04.2015, K.11672; locality 20, 12.12.2014, K.10978.

20. Helvella leucomelaena (Pers.) Nannf.: Among pine needles, locality 40, 29.03.2013, K.7831; locality 37, 20.03.2015, K.11436; locality 32, 12.04.2015, K.11741.

21. Helvella leucopus Pers.: Among grass under Populus sp. locality 21, 12.04.2015, K.11722.

22. Helvella macropus (Pers.) P. Karst.: Among grass in mixed forest, locality 11, 26.04.2014, K.8879.

23. Helvella monachella (Scop.) Fr.: Among grass in mixed forest, locality 27, 04.04.2015, K.11633.

24. Helvella queletii Bres.: Among leaf litter in mixed forest, locality 7, 28.03.2015, K.11542.

Morchellaceae Rchb. 
25. Mitrophora semilibera (DC.) Lév.: Among grass, locality 47, 05.04.2013, K.7857.

26. Morchella deliciosa Fr.: Among grass in pine forest, locality 56, 30.03.2013, K.7838; locality 50, 06.04.2013, K. 7867.

27. Morchella elata Fr.: Among grass in mixed forest, locality 27, 04.04.2015, K.11636; locality 33, 03.05.2015, K.11907.

28. Morchella esculenta (L.) Pers.: Among grass in mixed forest, locality 36, 03.04.2015, K.11574; locality 52, 17.04.2015, K.11758.

29. Verpa conica (O.F. Müll.) Sw.: Among grass under Populus sp., locality 62, 29.03.2015, K.11563.

Pezizaceae Dumort.

30. Iodophanus carneus (Pers.) Korf: On decaying dung, locality 48, 24.12.2014, K.11119.

31. Peziza badia Pers.: Among grass in mixed forest, locality 10, 08.11.2014, K. 10547.

32. Peziza cerea Sowerby ex Fr.: Among remains of herbs, locality 11, 01.11.2014, K.10458.

33. Peziza fimeti (Fuckel) E.C. Hansen: On cow dung, locality 9, 26.10.2014, K.10268.

34. Peziza pseudoviolacea Donadini: On ash in mixed forest, locality 11, 26.04.2014, K.8871.

35. Peziza vesiculosa Bull.: Among remains of herbs in mixed forest, locality 27, 04.04.2015, K.11637.

36. Peziza violacea Pers.: On ash, locality 18, 28.02.2015, K.11354.

37. Sarcosphaera coronaria (Jacq.) J. Schröt.: Among needle litter in mixed forest, locality 12, 27.04.2013, K.7919.

38. Terfezia boudieri Chatin: In soil among Helianthemum sp., locality 17, 30.03.2013, K.7839.

Pyronemataceae Corda

39. Cheilymenia fimicola (Bagl.) Dennis: On cow dung, locality 25, 09.03.2014, K.8606.

40. Cheilymenia theleboloides (Alb. \& Schwein.) Boud.: On decaying animal body parts, locality 7, 28.03.2015, K.11533.

41. Geopora arenicola (Lév.) Kers: On sandy soil among grass, locality 60, 20.04.2012, K.7418; locality 30, 05.12.2014, K.10873.

42. Geopora arenosa (Fuckel) S. Ahmad:
Among grass in Quercus sp. forest, locality 58, 12.04.2015, K.11704; locality 21, 04.04.2015, K.11617.

43. Geopora sumneriana (Cooke) M. Torre: Among grass in mixed forest, locality 20, 12.12.2014, K.10986.

44. Humaria hemisphaerica (F.H. Wigg.) Fuckel: Among grass in mixed forest, locality 38, 30.11.2014, K.10819.

45. Hypotarzetta insignis (Berthet \& Riousset) Donadini: On soil in pine forest, locality 35, 18.04.2015, K.11782.

46. Octospora grimmiae Dennis \& Itzerott: Among moss, locality 56, 01.03.2015, K.11369.

47. Tarzetta catinus (Holmsk.) Korf \& J.K. Rogers: On soil in mixed forest, locality 27, 04.04.2015, K.11627.

48. Tarzetta cupularis (L.) Svrček: On soil in mixed forest, locality 31, 18.04.2015, K.11770.

Tuberaceae Dumort.

49. Tuber borchii Vittad.: In soil under Quercus sp., locality 39, 24.10.2015, K.12541.

Sordariomycetes O.E. Erikss. \& Winka

Diaporthales Nannf.

Valsaceae Tul. \& C. Tul.

50. Valsa sordida Nitschke: On Populus sp. stump, locality 61, 09.03.2014, K.8550.

Hypocreales Lindau

Nectriaceae Tul. \& C. Tul.

51. Dialonectria episphaeria (Tode) Cooke:

On Diatrype sp. growing on Quercus sp. twigs, locality 36, 05.12.2014, K.10900; locality 37, 20.03.2015, K.11438.

Xylariales Nannf.

Diatrypaceae Nitschke

52. Diatrype bullata (Hoffm.) Fr.: On dead Fagus sp. twigs, locality 11, 12.04.2014, K.8837.

53. Diatrype disciformis (Hoffm.) Fr.: On dead Fagus sp. twigs, locality 11, 12.04.2014, K.8808.

54. Diatrype stigma (Hoffm.) Fr.: On dead Fagus sp. twigs, locality 12, 02.05.2014, K.8917.

Basidiomycota R.T. Moore

Agaricomycetes Doweld

Agaricales Underw.

Agaricaceae Chevall. 
55. Agaricus bitorquis (Quél.) Sacc.: Among grass, locality 49, 15.11.2015, K.12790.

56. Agaricus campestris L.: Meadow, locality 20, 05.12.2015, K.12810.

57. Agaricus moelleri Wasser: On soil in mixed forest, locality 26, 26.10.2014, K.10251.

58. Agaricus sylvaticus Schaeff.: Among grass in Quercus sp. forest, locality 36, 06.11.2015, K.12652.

59. Agaricus xanthodermus Genev.: Among grass in forest clearing, locality 56, 24.11.2013, K.6536.

60. Battarrea phalloides (Dicks.) Pers.: Among grass, locality 43, 05.04.2015, K.11666.

61. Bovista aestivalis (Bonord.) Demoulin: Among grass in pine forest, locality 26, 08.11.2015, K.12761.

62. Bovista nigrescens Pers.: Among grass, locality 36, 15.02.2015, K.8459.

63. Bovista plumbea Pers.: Among grass in mixed forest, locality 31, 18.04.2015, K.11772; locality 3, 18.04.2015, K.11774.

64. Coprinus comatus (O.F. Müll.) Pers.: Among grass, locality 56, 03.01.2015, K.11138; locality 36, 05.12.2014, K.10909; locality 41, 03.04.2015, K.11575.

65. Crucibulum laeve (Huds.) Kambly: On dead twigs, locality 25, 07.12.2013, K. 8457. 66. Cyathus olla (Batsch) Pers.: On woody particles and dungs, locality 38, 30.11.2014, K.10838; locality 34, 10.04.2015, K.11701.

67. Cyathus stercoreus (Schwein.) De Toni: On cow dung, locality 26, 26.10.2014, K.10263; locality 28, 12.12.2014, K.11048.

68. Cystoderma amianthinum (Scop.) Fayod: Among needle litter in pine forest, locality 28, 12.12.2014, K.11043.

69. Cystodermella cinnabarina (Alb. \& Schwein.) Harmaja: Among needle litter in pine forest, locality 49, 10.11.2013, K.8277.

70. Leucoagaricus leucothites (Vittad.) Wasser: Among grass near pine forest, locality $28,06.11 .2015$, K.12677; locality 23 , 06.11.2015, K.12696.

71. Lycoperdon excipuliforme (Scop.) Pers.: Among grass in mixed forest, locality 35 , 18.04.2015, K.11780.

72. Lycoperdon molle Pers.: Among grass in pine forest, locality 38, 29.11.2013, K.8372; locality 37, 06.11.2015, K.12657.
73. Lycoperdon perlatum Pers.: Among leaf-needle litter in mixed forest, locality 26 , 26.10.2014, K.10258; locality 37, 06.11.2015, K.12658.

74. Lycoperdon pratense Pers.: Among grass in Quercus sp. forest, locality 37, 06.11.2015, K.12649.

75. Macrolepiota excoriata (Schaeff.) Wasser: Among grass in Quercus sp. forest, locality 26, 26.10.2014, K.10264.

76. Macrolepiota mastoidea (Fr.) Singer: Among grass, locality 63, 02.12.2011, K.7336.

77. Macrolepiota procera (Scop.) Singer: Among grass in pine forest, locality 5, 18.10.2014, K. 9987.

78. Tulostoma brumale Pers.: Among grass with moss in Quercus sp. forest, locality 54, 07.03.2015, K.11400; locality 58, 12.04.2015, K.11712.

79. Tulostoma fimbriatum Fr.: Among grass, locality 57, 01.03.2015, K.11368; locality 43, 05.04.2015, K.11647.

80. Tulostoma melanocyclum Bres.: Among moss in mixed forest, locality 58, 20.03.2015, K.11439.

Amanitaceae R. Heim ex Pouzar

81. Amanita excelsa (Fr.) Bertill.: Among grass in Quercus sp. forest, locality 23, 06.11.2015, K.12692.

Bolbitiaceae Singer

82. Bolbitius titubans (Bull.) Fr.: Among grass under Populus and Salix sp., locality 62, 29.03.2015, K.11562.

83. Conocybe apala (Fr.) Arnolds: Among grass, locality 24, 03.12.2011, K.7351; locality 16, 14.12.2014, K.11090.

84. Conocybe filaris (Fr.) Kühner: On decaying cow dung, locality 28, 12.12.2014, K.11037.

85. Conocybe rickenii (Jul. Schäff.) Kühner: Among grass, locality 33, 03.05.2015, K.11905.

86. Galeropsis desertorum Velen. \& Dvořák: Among grass in mixed forest, locality 41, 06.11.2015, K.12667; locality 39, 07.11.2015, K.12718.

\section{Cyphellaceae Lotsy}

87. Chondrostereum purpureum (Pers.) Pouzar: On wood remains, locality 57, 10.11.2013, K. 8273.

Entolomataceae Kotl. \& Pouzar

88. Entoloma rusticoides (Gillet) Noordel.: 
Among grass in pine forest, locality 49, 15.11.2015, K.12802.

Hydnangiaceae Gäum. \& C.W. Dodge

89. Laccaria laccata (Scop.) Cooke: Among leaf and needle litter in mixed forest, locality 11, 01.11.2014, K.10406.

Hygrophoraceae Lotsy

90. Ampulloclitocybe clavipes (Pers.) Redhead, Lutzoni, Moncalvo \& Vilgalys: Among grass in pine forest, locality 24, 03.12.2011, K.7367.

91. Hygrocybe conica (Schaeff.) P. Kumm.: Among grass in mixed forest, locality 38 , 30.11.2014, K.10822.

Hymenogastraceae Vittad.

92. Galerina graminea (Velen.) Kühner: Among grass, locality 56, 30.11.2012, K.7634.

93. Galerina marginata (Batsch) Kühner: On dead twigs and stump in pine forest, locality 24, 03.12.2011, K.7358.

\section{Incertae Sedis}

94. Panaeolina foenisecii (Pers.) Maire: Among grass, locality 53, 24.10.2014, K.10096.

95. Panaeolus fimicola (Pers.) Gillet: Among grass in pine forest, locality 28, 12.12.2014, K.11035.

96. Panaeolus olivaceus F.H. Møller: Among grass, locality 33, 03.05.2015, K.11903.

Inocybaceae Jülich

97. Inocybe amblyospora Kühner: Among needle litter in pine forest, locality 4, 21.03.2014, K.8716.

98. Inocybe bongardii (Weinm.) Quél.: Among needle litter in pine forest, locality 34 , 10.04.2015, K.11699.

99. Inocybe cincinnata (Fr.) Quél.: Among needle litter in pine forest, locality 9, 21.03.2014, K.8726.

100. Inocybe geophylla (Bull.) P. Kumm.: Among leaf-needle litter in mixed forest, locality 38, 30.11.2014, K.10820.

101. Inocybe rimosa (Bull.) P. Kumm.: Among grass in mixed forest, locality 12 , 30.11.2013, K.8377.

102.Phaeomarasmius erinaceus (Fr.)

Scherff. ex Romagn.: On Quercus sp. branch, locality 12, 01.11.2014, K.10399.

Marasmiaceae Roze ex Kühner

103. Calyptella capula (Holmsk.) Quél.: On Quercus sp. twigs, locality 36, 05.12.2014,
K.10927.

104. Henningsomyces candidus (Pers.)

Kuntze: On pine stump, locality 4, 14.11.2014, K.10660.

105. Macrocystidia cucumis (Pers.) Joss.: Among leaf litter in pine forest, locality 56, 30.11.2012, K.7636.

106. Marasmius anomalus Lasch ex Rabenh.: Among grass, locality 6, 18.10.2014, K.9925; locality 26, 14.12.2014, K.11101.

107. Marasmius epodius Bres.: Among grass in pine forest, locality 30, 05.12.2014, K.10866.

108. Marasmius oreades (Bolton) Fr.: Among grass, locality 12, 02.05.2014, K.8903.

109. Marasmius wynneae Berk. \& Broome: Among leaf litter under Quercus sp., locality 61, 02.12.2012, K. 7346.

Mycenaceae Roze

110. Hemimycena lactea (Pers.) Singer: On decaying needle litter, locality 36, 05.12.2014, K.10910; locality 24, 03.12.2011, K.7351.

111. Mycena crocata (Schrad.) P. Kumm.: On decaying wood remains, locality 11, 14.11.2015, K.12783.

112. Mycena pura (Pers.) P. Kumm.: Around Quercus sp., locality 26, 07.03.2014, K.8499. 113. Mycena rosea Gramberg: Among needle litter in pine forest, locality 26, 26.10.2014, K.10259.

114. Mycena seynii Quél.: On pine cones, locality $57,16.11 .2012$, K.7508; locality 36 , 05.12.2014, K.10922.

115. Scytinotus violaceofulvus (Batsch) Courtec.: On Abies sp. twigs, locality 11, 12.04.2014, K.8800.

116.Xeromphalina campanella (Batsch) Kühner \& Maire: On dead stump, locality 37, 18.04.2010, K.7171.

117. Xeromphalina cauticinalis (With.) Kühner \& Maire: Among needle litter, locality 3, 30.11.2013, K.8400.

Niaceae Jülich

118. Lachnella alboviolascens (Alb. \& Schwein.) Fr.: On dead Populus sp. twigs, locality 51, 06.12.2014, K.10968.

Omphalotaceae Bresinsky

119. Gymnopus dryophilus (Bull.) Murrill: Among leaf-needle litter in mixed forest, locality 28, 06.11.2015, K.12669; locality 39, 07.11.2015, K.12719. 
120. Gymnopus erythropus (Pers.) Antonín, Halling \& Noordel.: Among decaying Quercus sp. leaves, locality 36, 05.12.2014, K.10896.

121. Gymnopus ocior (Pers.) Antonín \& Noordel.: Among leaf-needle litter in mixed forest, locality 56, 16.11.2012, K.7507.

122. Gymnopus quercophilus (Pouzar) Antonín \& Noordel.: Among decaying Quercus sp. leaves, locality 37, 10.04.2015, K.11689.

123. Omphalotus olearius (DC.) Singer: On Quercus sp. stump, locality 525, 07.11.2015, K.12699.

Physalacriaceae Corner

124. Armillaria borealis Marxm. \& Korhonen: On decaying stump, locality 59, 16.02.2014, K.8497.

125. Armillaria mellea (Vahl) P. Kumm.: On decaying stump, locality 24, 03.12.2011, K.7370.

126. Strobilurus stephanocystis (Kühner \& Romagn. ex Hora) Singer: On pine cones, locality 24, 03.12.2011, K.7360.

127. Strobilurus tenacellus (Pers.) Singer: Among needle litter in pine forest, locality 24 , 03.12.2011, K.7368.

Pleurotaceae Kühner

128. Hohenbuehelia petaloides (Bull.) Schulzer: Among leaf litter around Quercus sp., locality 1, 26.12.2009, K.6921; among needle litter, locality 24, 03.12.2011, K.7385. 129. Pleurotus dryinus (Pers.) P. Kumm.: On Salix sp. stump, locality 62, 02.11.2014, K.10512.

130. Pleurotus ostreatus (Jacq.) P. Kumm.: On Salix sp. stump, locality 42, 13.04.2013, K.7896; locality 20, 12.04.2013, K.7875.

Pluteaceae Kotl. \& Pouzar

131. Pluteus romellii (Britzelm.) Sacc.: On soil around Salix sp., locality 62, 19.05.2013, K.8450.

132. Volvopluteus gloiocephalus (DC.) Justo: Among grass, locality 47, 25.11.2012, K.7620.

Psathyrellaceae Vilgalys, Moncalvo \& Redhead

133. Coprinellus disseminatus (Pers.) J.E. Lange: On damp soil under Populus sp., locality 46, 13.04.2013, K.7880; locality 43, 13.04.2013, K.7894.

134. Coprinellus ephemerus (Bull.) Redhead, Vilgalys \& Moncalvo: Among grass in pine forest clearing, locality 25, 09.03.2014, K.8607; locality 19, 22.03.2015, K.11493.

135. Coprinellus micaceus (Bull.) Vilgalys, Hopple \& Jacq. Johnson: On Populus sp. stump, locality 45, 30.03.2013, K.7844; locality 46, 23.11.2013, K.8317.

136. Coprinopsis atramentaria (Bull.) Redhead, Vilgalys \& Moncalvo: Around Populus sp. stump, locality 21, 12.04.2015, K.11723.

137. Coprinopsis lagopides (P. Karst.) Redhead, Vilgalys \& Moncalvo: On cow dung, locality 521, 18.04.2015, K.11764.

138. Coprinopsis nivea (Pers.) Redhead, Vilgalys \& Moncalvo: On decaying cow dung, locality 31, 18.04.2015, K.11767.

139. Parasola plicatilis (Curtis) Redhead, Vilgalys \& Hopple: Among grass under Salix sp., locality 13, 25.10.2014, K.10220.

140. Psathyrella bipellis (Quél.) A.H. Sm.: Among leaf litter in mixed forest, locality 22, 12.12.2014, K.10990.

141. Psathyrella candolleana (Fr.) Maire: Around Populus sp. remains, locality 42, 21.04.2012, K.7439; locality 33, 03.05.2015, K.11906.

Pterulaceae Corner

142. Pterula multifida (Chevall.) Fr.: Among needle litter in pine forest, locality 28, 05.12.2015, K.12817.

Schizophyllaceae Quél.

143. Schizophyllum amplum (Lév.) Nakasone: On dead Populus sp. twigs, locality 15, 25.10.2014, K.10195.

144. Schizophyllum commune Fr.: On stump, locality $25,29.03 .2013$, K.7822; locality 36 , 15.02.2014; K.8462.

Strophariaceae Singer \& A.H. Sm.

145.Agrocybe molesta (Lasch) Singer: Among grass, locality 53, 24.10.2014, K.10095.

146. Agrocybe pediades (Fr.) Fayod: Among grass in pine forest clearing, locality 32 , 12.04.2015, K. 11738.

147. Agrocybe vervacti (Fr.) Singer: Among grass, locality 49, 15.11.2015, K.12801.

148. Cyclocybe cylindracea (DC.) Vizzini \& Angelini: On Populus sp. stump, locality 44, 30.03.2013, K.7842; locality 26, 08.11.2015, K.12756; locality 14, 08.03.2014, K.8549.

149. Hypholoma fasciculare (Huds.) P. Kumm.: Aroung Quercus sp. stump, locality 36, 05.12.2014, K.10921. 
150. Protostropharia semiglobata (Batsch) Redhead, Moncalvo \& Vilgalys: Among grass, locality 49, 15.11.2015, K.12787.

151. Psilocybe coprophila (Bull. : Fr.) P. Kumm: On decaying cow dung, locality 25, 29.03.2013, K.7821; locality 19, 22.03.2015, K.11499.

152. Stropharia aeruginosa (Curtis) Quél.: Among grass in mixed forest, locality 23, 06.11.2015, K.12686; locality 40, 07.11.2015, K.12704.

153. Stropharia coronilla (Bull.) Quél.: Among grass in pine or mixed forest, locality $29, \quad 19.10 .2014, \quad$ K.10021; locality 30, 05.12.2014, K.10857.

Tricholomataceae R. Heim ex Pouzar 154. Arrhenia retiruga (Bull.) Redhead: Among moss in pine forest, locality 61, 29.03.2015, K.11549.

155. Arrhenia rickenii (Hora) Watling: Among moss in Quercus sp. forest, locality 30, 05.12.2014, K.10882.

156. Arrhenia spathulata (Fr.) Redhead: On moss, locality 58, 12.04.2015, K.11717; locality 20 , 06.11.2015, K.12683; locality 38 , 30.11.2014, K.10812.

157. Clitopaxillus alexandri (Gillet) G. Moreno, Vizzini, Consiglio \& P. Alvarado: Among needle litter in pine forest, locality 24 , 03.12.2011, K.7362.

158. Clitocybe gibba (Pers.) P. Kumm.: Among needle litter in pine forest, locality 36 , 05.12.2014, K.10929.

159. Infundibulicybe geotropa (Bull.) Harmaja: Among leaf litter in Quercus sp., locality 23, 06.11.2015, K.12688.

160. Lepista nuda (Bull.) Cooke: Among leaf or needle litter in pine, Quercus or mixed forest, locality 57, 30.03.2013, K.7837; locality 26, 28.03.2015, K.11528.

161. Leucopaxillus gentianeus (Quél.) Kotl.: Among needle litter in pine forest, locality 11 , 14.11.2015, K.12786.

162. Melanoleuca cognata (Fr.) Konrad \& Maubl.: Among grass in mixed forest, locality 3, 18.04.2015, K.11775.

163. Melanoleuca exscissa (Fr.) Singer: Among grass in pine forest clearing, locality 37, 10.04.2015, K.11690.

164. Melanoleuca polioleuca (Fr.) Kühner \& Maire: Among grass in mixed forest, locality 36, 05.12.2014, K.10920; locality 28, 12.12.2014, K.11041.
165. Melanoleuca stridula (Fr.) Singer: Among needle litter in pine forest, locality 26 , 07.03.2014, K.8504.

166. Myxomphalia maura (Fr.) H.E. Bigelow: Among needle litter, locality 56, 16.11.2012, K.7501.

167. Pseudoclitocybe cyathiformis (Bull.: Fr.) Singer: Among needle litter in pine forest, locality 20, 29.11.2014, K.10763; locality 30 , 05.12.2014, K.10878.

168. Resupinatus trichotis (Pers.) Singer: On Quercus sp. twigs, locality 27, 12.12.2014, K.11025.

169. Tricholoma terreum (Schaeff.) P. Kumm.: Among needle litter in pine forest, locality 56, 16.11.2012, K.7506; locality 57, 21.03.2014, K. 8689; locality 36, 05.12.2014, K.10916.

\section{Tubariaceae Vizzini}

170. Tubaria conspersa (Pers.) Fayod: Among leaf litter, locality 32, 31.03.2013, K.7852.

Typhulaceae Jülich

171. Typhula fistulosa (Holmsk.) Olariaga: On herb remains, locality 10, 08.11.2014, K.10554.

Auriculariales J. Schröt.

Auriculariaceae Fr.

172. Exidia glandulosa (Bull.) Fr.: On dead Quercus sp. twigs, locality 39, 12.04.2015, K.11742.

173. Exidia nigricans (With.) P. Roberts: On dead Quercus sp. twigs, locality 20, 12.12.2014, K.11004.

Boletales E.-J. Gilbert

Boletaceae Chevall.

174. Boletus edulis Bull.: Among grass in mixed forest, locality 11, 24.10.2014, K.10183.

175. Boletus erythropus Pers.: Among grass in Quercus sp. forest, locality 39, 07.11.2015, K.12722.

176.Xerocomellus chrysenteron (Bull.) Sutara: Among grass in mixed forest, locality 36, 06.11.2015, K.12695; locality 39, 07.11 .2015 , K.12722.

Diplocystidiaceae Kreisel

177. Astraeus hygrometricus (Pers.) Morgan: Among leaf litter in mixed forest, locality 22, 12.12.2014, K.10995; locality 27, 04.04.2015, K.11632

Gomphidiaceae Maire ex Jülich 178. Chroogomphus rutilus (Schaeff.) O.K. 
Mill.: Among needle litter in pine forest, locality $57,30.11 .2012$, K.7626; locality 22 , 29.11.2014, K.10767.

Rhizopogonaceae Gäum. \& C.W. Dodge 179. Rhizopogon luteolus Fr.: In soil in pine forest, locality 22, 29.11.2014, K.10765; locality 19, 22.03.2015, K.11498; locality 41, 03.04.2015, K.11577.

180. Rhizopogon roseolus (Corda) Th. Fr.: In soil in pine forest, locality 30, 05.12.2014, K.10875; locality 32, 12.04.2015, K.11737.

Sclerodermataceae Corda

181. Pisolithus arhizus (Scop.) Rauschert: Among leaf litter in Quercus sp. forest, locality 39, 24.10.2015, K.12542.

182. Scleroderma cepa Pers.: Among grass in mixed forest, locality 4, 14.11.2014, K. 10631.

183. Scleroderma verrucosum (Bull.) Pers.: Among grass, locality 51, 13.11.2015, K.12778.

Suillaceae Besl \& Bresinsky

184. Suillus collinitus (Fr.) Kuntze: Among needle litter in pine forest, locality 34 , 10.04.2015, K.11697; locality 32, 12.04.2015, K.11736.

185. Suillus granulatus (L.) Roussel: Among grass in mixed forest, locality 27, 04.04.2015, K.11638.

186. Suillus luteus (L.) Roussel: Among needle litter in pine forest, locality 56, 16.11.2012, K.7495.

Tapinellaceae C. Hahn

187. Tapinella panuoides (Fr.) E.-J. Gilbert: On decaying stump, locality 24, 03.12.2011, K.7405.

Cantharellales Gäum

Clavulinaceae Donk

188. Clavulina cinerea (Bull.) J. Schröt.: Among needle litter in pine forest, locality 12 , 14.11.2014, K. 10648.

Geastrales K. Hosaka \& Castellano

Geastraceae Corda

189. Geastrum minimum Schwein.: On mossy soil in pine forest, locality 28 , 12.12.2014, K.11044.

190. Geastrum pectinatum Pers.: Among needle litter in pine forest, locality 12 , 02.05.2014, K.8922.

191.Sphaerobolus stellatus Tode: On Quercus remain, locality 36, 05.12.2014, K.10902.

Gomphales Jülich
Gomphaceae Donk

192. Gautieria trabutii (Chatin) Pat.: In soil under Quercus sp., locality 151, 02.05.2014, K.8912.

Hymenochaetales Oberw.

Hymenochaetaceae Imazeki \& Toki

193. Phellinus hartigii (Allesch. \& Schnabl) Pat.: On Abies sp. stump, locality 63, 13.04.2013, K. 7897.

194. Phellinus pomaceus (Pers.) Maire: On Amygdalus sp. stump, locality 26, 14.12.2014, K.11116.

Phallales E. Fisch.

Phallaceae Corda

195. Phallus impudicus L.: Among grass in mixed forest, locality 4, 14.11.2014, K.10635.

Polyporales Gäum.

Ganodermataceae Donk

196. Ganoderma adspersum (Schulzer) Donk: On stump, locality 43, 13.04.2013, K.7895.

197. Ganoderma lucidum (Curtis) P. Karst.:

On decaying stump, locality 46, 13.04.2013, K.7885; locality 11, 12.04.2014, K.8834.

Meruliaceae Rea

198. Bjerkandera adusta (Willd.) P. Karst.:

On Quercus sp. stump, locality 21, 12.04.2015, K.11720.

Phanerochaetaceae Jülich

199. Terana coerulea (Lam.) Kuntze: On Quercus sp. twigs, locality 20, 12.12.2014, K.10979.

Polyporaceae Fr. ex Corda

200. Fomes fomentarius (L.) Fr.: On Salix sp. stump, locality 46, 13.04.2013, K. 7889.

201. Lentinus arcularius (Batsch) Zmitr.: On dead twigs, locality A2, 24.02.2010, K. 7101. 202. Lentinus brumalis (Pers.) Zmitr.: On dead twigs, locality 20, 31.03.2013, K.7846.

203. Lentinus tigrinus (Bull.) Fr.: Around Populus sp. stump, locality 46, 21.04.2014, K.7441.

204. Lenzites betulina (L.) Fr.: On pine stump, locality 3, 18.04.2015, K.11773.

205. Royoporus badius (Pers.) A.B. De: On dead Fagus sp. twigs, locality 11, 01.11.2014, K.10457.

206. Trametes hirsuta (Wulfen) Lloyd: On dead Quercus sp. twigs, locality 57, 30.03.2013, K.7838; locality 7, 28.03.2015, K.11539; locality 58, 12.04.2015. K.11715.

207. Trametes versicolor (L.) Lloyd: On Quercus sp. stump, locality 55, 12.04.2015. 
K.11716; locality 3, 18.04.2015, K.11777.

Russulales Kreisel ex P.M. Kirk, P.F. Cannon \& J.C. David

Auriscalpiaceae Maas Geest.

208. Lentinellus micheneri (Berk. \& M.A. Curtis) Pegler: Among needle litter in pine forest, locality 24, 03.12.2011, K.7353.

\section{Russulaceae Lotsy}

209. Lactarius deliciosus (L.) Gray: Among needle litter in mixed forest, locality 24 , 02.12.2011, K.7356; locality 22, 29.11.2014, K.10766.

210. Lactarius torminosus (Schaeff.) Gray: Among grass in Quercus sp. forest, locality 40, 07.11.2015, K.12710.

211. Russula virescens (Schaeff.) Fr.: Among needle litter in mixed forest, locality 12 , 01.11.2014, K.10376.

Stereaceae Pilát

212. Stereum hirsutum (Willd.) Pers.: On dead Quercus sp. twigs, locality 41, 31.03.2013, K.7845; locality 22, 12.12 .2014 , K.11003; locality 36, 04.04.2015, K.11603.

Thelephorales Corner ex Oberw.

Bankeraceae Donk

213. Hydnellum caeruleum (Hornem.) P. Karst.: Among needle litter in pine forest, locality 24, 03.12.2011, K.7372.

Thelephoraceae Chevall.

214. Thelephora terrestris Ehrh.: Among needle litter in pine forest, locality 38, 18.04.2010, K.7170.

Dacrymycetes Doweld

Dacrymycetales Henn.

Dacrymycetaceae J. Schröt.
215. Calocera cornea (Batsch) Fr.: On dead Quercus sp. stump, locality 11, 01.11.2014, K.10440.

216.Dacrymyces capitatus Schwein.: On Quercus sp. twigs, locality 36, 05.12.2014, K.10907.

217. Dacrymyces stillatus Nees: On Quercus sp. twigs, locality 19, 22.03.2015, K.11480.

\section{Discussion and Conclusion}

Two hundred and seventeen macrofungi species belonging to 129 genera, 63 families, 19 orders and 6 classes were determined from Gaziantep. 54 of them belong to Ascomycota (1 Diaporthales, 13 Helotiales, 1 Hypocreales, 1 Patellariales, 33 Pezizales, 3 Xylariales) and 163 to Basidiomycota (117 Agaricales, 2 Auriculariales, 14 Boletales, 1 Cantharellales, 3 Dacrymycetales, 3 Geastrales, 1 Gomphales, 2 Hymenochaetales, 1 Phallales, 12 Polyporales, 5 Russulales, 2 Thelephorales).

Regarding the determined 217 species, Agaricaceae, Tricholomataceae and Pyronemataceae were found to be the most crowded three families in the region with 26 , 16 and 10 species respectively.

There are some similarities between the findings of the present study and those of previous studies. The comparative data between the macrofungal composition of Gaziantep and the studies conducted in surrounding provinces was given in Table 2 .

Table 2. Similarity percentages of neighboring studies with Gaziantep province.

\begin{tabular}{lccc}
\hline & $\begin{array}{c}\text { Number of } \\
\text { identical taxa }\end{array}$ & $\begin{array}{c}\text { Total } \\
\text { taxa }\end{array}$ & $\begin{array}{c}\text { Similarity } \\
\text { percentage (\%) }\end{array}$ \\
\hline Adıyaman (Kaya, 2009a) & 98 & 222 & 44.14 \\
Hatay (Baba et al., 2013, 2014, Güngör et al., 2016) & 63 & 153 & 41.18 \\
Kahramanmaraş (Kaya, 2009b) & 106 & 337 & 31.45 \\
Kilis (Solak et al., 2014) & 25 & 52 & 42.31 \\
Osmaniye (Günay \& Demirel 2006, Solak et al., 2011) & 40 & 87 & 41.38 \\
Şanliurfa (Kaya, 2015) & 89 & 118 & 66.95 \\
\hline
\end{tabular}

Sixty of the 217 taxa are edible, but only six species, Agaricus campestris, Coprinus comatus, Lactarius deliciosus, Pleurotus ostreatus, Terfezia boudieri and Tricholoma terreum, are collected and consumed in the region by local people. Among them, Terfezia boudieri have local economic importance while $T$. anatolicum have international economic importance. During autumn, lots of T. anatolicum fruit bodies are collected by some villagers for exporting companies.

One hundred and thirty eight of the determined taxa are regarded as inedible, while 19 are more or less poisonous. Among 
the determined taxa, 60 are wood decaying, 6 are hygogeous, 7 are coprophilous, 2 are pyrophilous and 5 are bryophilous.

With the addition of 217 species to the previously reported 187 taxa, the determined macrofungi number in Gaziantep increased to 404.

\section{Acknowledgements}

This work was financed by the Scientific and Technical Research Council of Turkey (TUBITAK 212 T 112).

\section{References}

Akman, Y. (1999). İklim ve Biyoiklim. Ankara, Kariyer Matbacilık Ltd.

Baba, H., Alkan, S. \& Kaşı1k, G. (2013). Macrofungi of Antakya (Hatay) and its Environment. Mantar Dergisi, 4(1), 11-20.

Baba, H., Alkan, S. \& Kaşık, G. (2014). Macrofungi of Mustafa Kemal University Tayfur Sökmen Campus (Hatay- Turkey) and environment. Mantar Dergisi, 5(2), 1-8.

Bessette, A. E., Bessette, A. R. \& Fischer, D. W. (1997). Mushrooms of Northeastern North America. Syracuse, NY, USA, Syracuse University Press.

Bessette, A. E., Roody, W. C. \& Bessette, A. R., Dunaway, D.L. (2007). Mushrooms of the Southeastern United States. Syracuse University Press.

Beug, M. W., Bessette, A. E. \& Bessette, A. R. (2014). Ascomycete Fungi of North America. Austin, Texas University Press.

Breitenbach, J. \& Kränzlin, F. (1984-2000). Fungi of Switzerland, Volumes 1-5. Lucerne, Verlag Mykologia.

Demirel, K., Uzun, Y., Keleş, A., Akçay, M. E. \& Acar, İ. (2017). Macrofungi of Karagöl-Sahara National Park (Artvin/Turkey). Biological Diversity and Conservation, 10(2), 32-40.

Ellis, M. B. \& Ellis, J. P. (1990). Fungi without gills (Hymenomycetes and Gasteromycetes). An identification handbook, Landon, Chapman and Hall.

Ellis, M. B. \& Ellis, J. P. (1997). Microfungi on Land Plants. An Identicication Handbook. UK, Richmond Publishing.

Günay, N. \& Demirel, K. (2006). Düziçi ve Bahçe (Osmaniye) Yöresinde Yetişen Makrofunguslar Üzerinde Taksonomik Bir Araștırma. Yüzücü Yll Üniversitesi Fen Bilimleri Enstitüsü Dergisi, 11(1), 17-24.

Güngör, H., Solak, M. H., Allı, H., Işıloğlu, M. \& Kalmış, E. (2016). Contributions to the macrofungal diversity of Hatay province,
Turkey. Biological Diversity and Conservation, 9(1): 101-106.

Hansen, K. \& Pfister, D. H. (2006). Systematics of the Pezizomycetes -the operculate discomycetes. Mycologia 98(6), 1029-1040.

Index Fungorum. (2018). http://www.indexfungorum.org/names/Names. asp / [30 August 2018]

Işık, H. \& Türkekul, İ. (2017). A new record for Turkish mycota from Akdağmadeni (Yozgat) province: Russula decolorans (Fr.) Fr. Anatolian Journal of Botany, 1(1), 1-3.

İskender, E., Zeynalov, Y., Özaslan, M., Çakır, B. M., Yayla, F. \& İncik, F. N. (2005). Tree and shrub species of the Huzurlu High Plateau (Gaziantep, Turkey). Phytologia Balcanica, 11(2), 149-156.

Kaşık, G., Aktaş, S., Alkan, S. \& Öztürk, C. (2017). Selçuk Üniversitesi Alaeddin Keykubat Kampüsü (Konya) mantarlarına ilaveler. Mantar Dergisi, 8(2), 129-136.

Kaya, A. (2009a). Macrofungal diversity of Adiyaman Province (Turkey). Mycotaxon, 110, 43-46.

Kaya, A. (2009b). Macromycetes of Kahramanmaraş Province (Turkey). Mycotaxon, 108, 31-34.

Kaya, A. (2015). Contributions to the macrofungal diversity of Atatürk Dam Lake Basin. Turkish Journal of Botany, 39(1), 162-172.

Kaya, A. \& Uzun, Y. (2015). Six new genus records for Turkish Pezizales from Gaziantep Province. Turkish Journal of Botany, 39(3), 506-511.

Kaya, A., Uzun, Y., Karacan, İ. H. \& Yakar, S. (2016). Contributions to Turkish Pyronemataceae from Gaziantep Province. Turkish Journal of Botany, 40(3), 298-307.

Kirk, P. M., Cannon, P. F., Minter, D. W. \& Stalpers, J. A. (2008). Dictionary of the Fungi. 10th ed. Wallingford, UK, CAB International.

Lukić, N. (2009). The distribution and diversity of Boletus genus in Central Serbia. Kragujevac J. Sci., 31, 59-68.

Medardi, G. (2006). Ascomiceti d'Italia. A.M.B. Fonzazione Centro Studio Micologici.

Miller, O. K. \& Miller, H. H. (1988). Gasteromycetes. USA, Mad River Press.

Öztürk, C., Pamukçu, D. \& Aktaş, S. (2017). Nallıhan (Ankara) ilçesi Makrofungusları. Mantar Dergisi, 8(1), 60-67.

Philips, R. (1981). Mushrooms and other Fungi of Great Britain and Europe. Pan Books.

Sesli, E. (2018). Cortinarius ve Lyophyllum cinslerine ait yeni kayitlar. Mantar Dergisi, 9(1), 18-23.

Sesli, E. \& Denchev, C. M. (2014). Checklists of the myxomycetes, larger ascomycetes, and 
larger basidiomycetes in Turkey. 6th edn. Mycotaxon Checklists Online (http,//www.mycotaxon.com/resources/checkl ists/sesli-v106-checklist.pdf),1-136.

Solak, M. H., Allı, H. \& Işıloğlu, M. (2011). Macrofungi of Osmaniye Province. Mantar Dergisi, 2(1-2), 1-7.

Solak, M. H., Allı, H., Işıloğlu, M., Güngör, H. \& Kalmış, E. (2014). Contributions to the macrofungal diversity of Kilis Province. Turkish Journal of Botany, 38(1), 180-185.

Solak, M. H., Işıloğlu, M., Kalmış, E. \& Allı, H. (2015). Macrofungi of Turkey, Checklist. Volume-II. Bornova, Üniversiteliler Ofset.

Şelem, E., Keleş, A., Acar, İ. \& Demirel, K. (2019). Edible macrofungi determined in Gürpınar (Van) district. Anatolian Journal of Botany, 3(1), 7-12.

Tırpan, E., Çöl, B., Şen, İ. \& Allı, H. (2018). Macrofungi of Datça Peninsula (Turkey).
Biological Diversity and Conservation, 11(3), 90-98.

Türkekul, İ. \& Işık, H. (2017). Bozatalan (Tokat) Yöresi Makrofungusları. Kafkas Üniversitesi Fen Bilimleri Enstitüsü Dergisi, 9(1), 5-11.

Uzun, Y., Karacan, İ. H., Yakar, S. \& Kaya, A. (2018a). New additions to Turkish Tricholomataceae. Anatolian Journal of Botany, 2(2), 65-69.

Uzun, Y., Yakar, S., Karacan, İ.H. \& Kaya, A. (2018b). New additions to the Turkish Pezizales. Turkish Journal of Botany, 42(3), 335-345.

Uzun, Y., Kaya, A., Karacan, İ.H., Kaya, Ö.F. \& Yakar, S. (2015). Macromycetes determined in Islahiye (Gaziantep) district. Biological Diversity and Conservation, 8(3), 209-217. 